\section{Large Dehisced Senile Calcific Plaque Associated with Necro- tizing Scleritis and Perforation}

\section{William McSwain*, Roxanna Ursea and Le Yu}

Department of Ophthalmology and Vision Science, University of Arizona, USA

\begin{abstract}
\section{Background}

Senile calcific plaques are commonly found in elderly patients, but are rarely discussed in medical literature because they are almost always benign and can be observed.

\section{Case presentation}

We report a case of an elderly patient with a painful dehisced senile calcific plaque with associated corneal thinning and scleral perforation secondary to necrotizing scleritis. The patient was initially referred to our clinic to have an enucleation. But her eye was saved by removing the dehisced plaque and closing the defect with a combination of scleral patch grafting and amniotic membrane transplantation.

\section{Discussion}

Necrotizing scleritis may be associated with dehiscence of a senile calcific plaque and scleral perforation. These difficult cases may be managed using scleral patch grafting combined with amniotic membrane transplant.

Keywords: Cornea; Sclera; Scleritis
\end{abstract}

\section{Introduction}

Senile calcific plaques are very common in elderly patients and occur at the insertions of the horizontal rectus muscles. Histologically, they consist of a core of calcium surrounded by a rim of acellular collagen [1]. It is thought that they form due to scleral dehydration, actinic solar damage, or mechanical stress exerted on the sclera by the horizontal rectus muscles [2]. Most often, they are benign and can be observed. But there have been rare reports of scleral perforation associated with plaque dehiscence $[3,4]$.

${ }^{*}$ Corresponding author: William McSwain, Department of Ophthalmology and Vision Science, University of Arizona, $655 \mathrm{~N}$ Alvernon Way, Suite 108 Tucson, AZ 85711, USA, Tel: +1 5207899439; Fax: +1 5203213665; E-mail: william.h.mcswain@gmail.com

Citation: McSwain W, Ursea R, Yu L (2015) Large Dehisced Senile Calcific Plaque Associated with Necrotizing Scleritis and Perforation. J Ophthalmic Clin Res 2: 013

Received: May 08, 2015; Accepted: September 15, 2015; Published: September 29, 2015
Necrotizing scleritis is an inflammatory process that may cause destruction of the sclera and globe perforation. Thinning of the sclera causes it to appear bluish-gray as the underlying uvea becomes visible [5]. Surgical management of perforation due to necrotizing scleritis is difficult. Options include patch grafting with fascia lata, processed pericardium, dermal, and scleral patch grafts. Enucleation may be the only option if the defect is too large and cannot be repaired [6-9].

We report a case of an elderly patient with a painful dehisced senile calcific plaque with associated corneal thinning and scleral perforation secondary to necrotizing scleritis.

\section{Case Presentation}

A 90-year-old Caucasian woman with a history of possible rheumatoid arthritis presented with a large growth on her right eye associated with severe pain, photophobia, and progressive vision loss over a period of 6 months. Ocular history was significant for pseudophakia and nonexudative macular degeneration in both eyes. A $30 \mathrm{lb}$ unintentional weight loss, non-healing skin lesions over her nose, and lower back pain were noted over the past year. The best corrected visual acuity was 20/400 in the right eye and 20/60 in the left eye. The right eye appeared hypotonus with anterior chamber shallowing and corneal striae (Figure 1a). Slit lamp examination showed a large extruded calcified mass on the temporal side of the right eye that had eroded through the conjunctiva (Figure 1b). The sclera adjacent and superonasal to the calcified mass was very thin, but no leak was visualized with sodium fluorescein (Figure 1c). Significant perilimbal corneal thinning was observed adjacent to the thinned sclera from 8:00 to 12:00 oclock (Figure 1d). Intraocular pressure by applanation was $3 \mathrm{mmHg}$ in the right eye and $13 \mathrm{mmHg}$ in the left eye. Ultrasonic biomicroscopy revealed severe scleral thinning deep and posterior to the calcific mass (Figure 2).

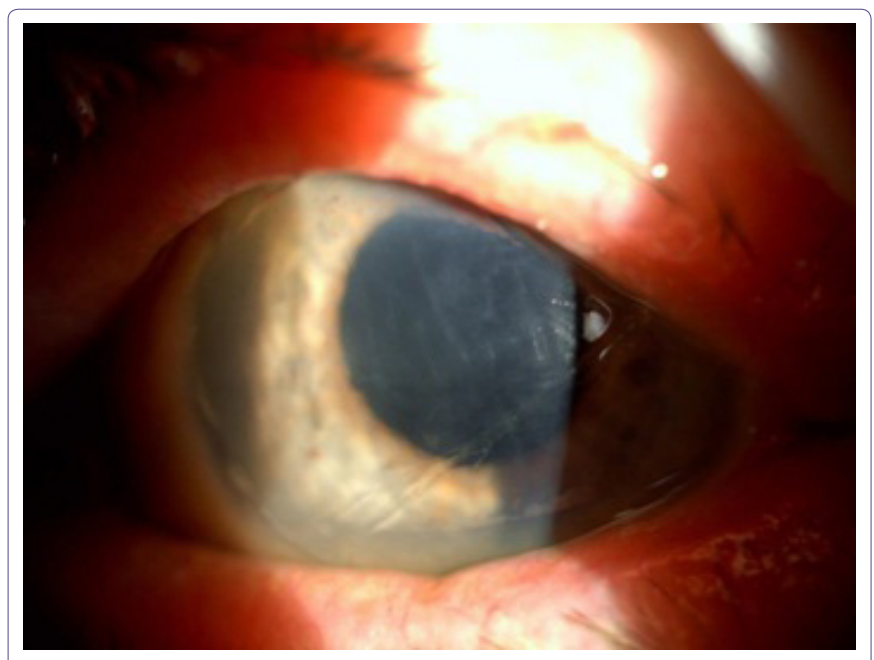

Figure 1a: Anterior chamber shallowing and corneal striae.

Systemic workup did not identify an underlying cause of the necrotizing scleritis. After informed consent was obtained, the patient 


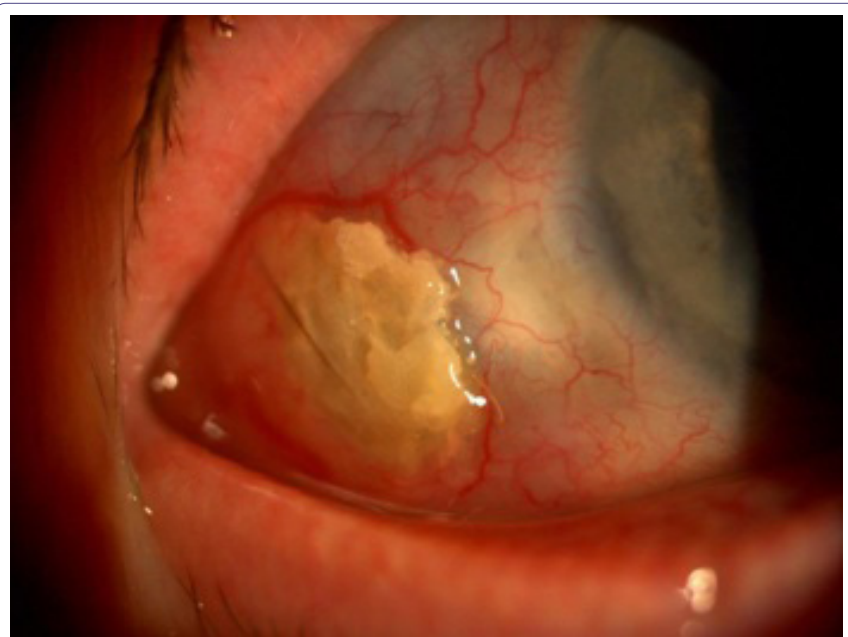

Figure 1b: Calcific mass extruding through conjunctiva.

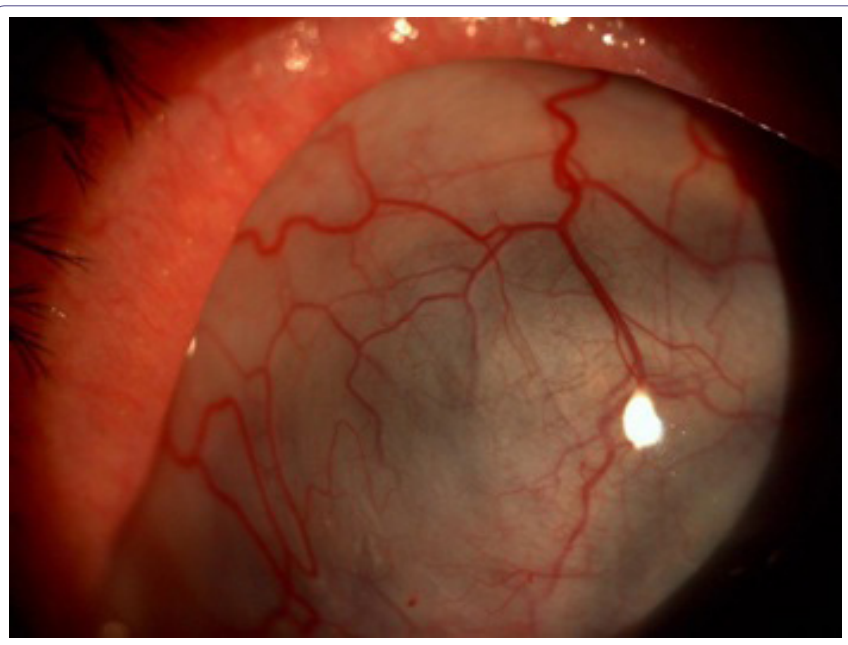

Figure 1c: Scleral thinning adjacent to mass.

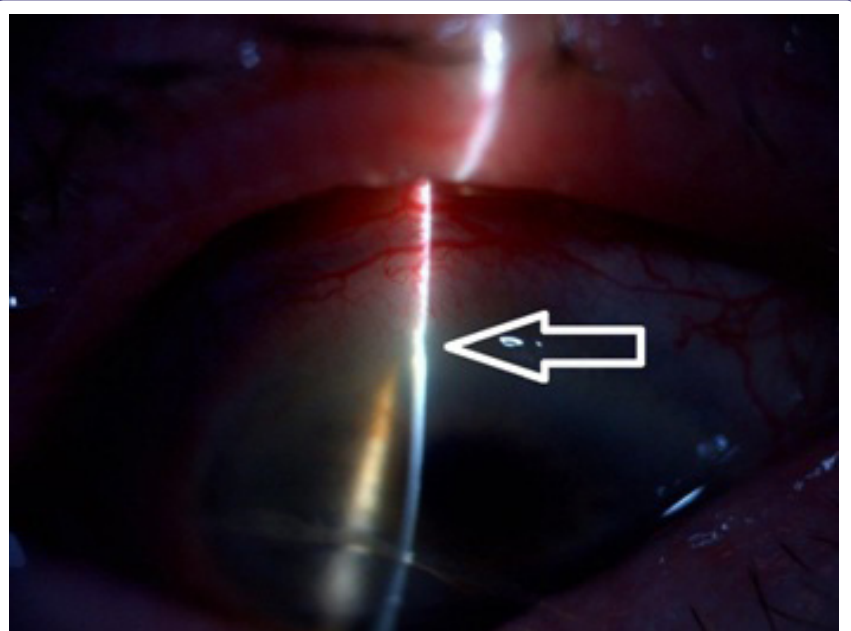

Figure 1d: Perilimbal corneal thinning from 8:00 to 12:00 o'clock.

was taken to the operating room for removal of the extruded senile calcific mass and repair of the scleral perforation.

The conjunctival mass was carefully dissected and excised from the sclera. The mass was much larger than its visible size on slit lamp exam

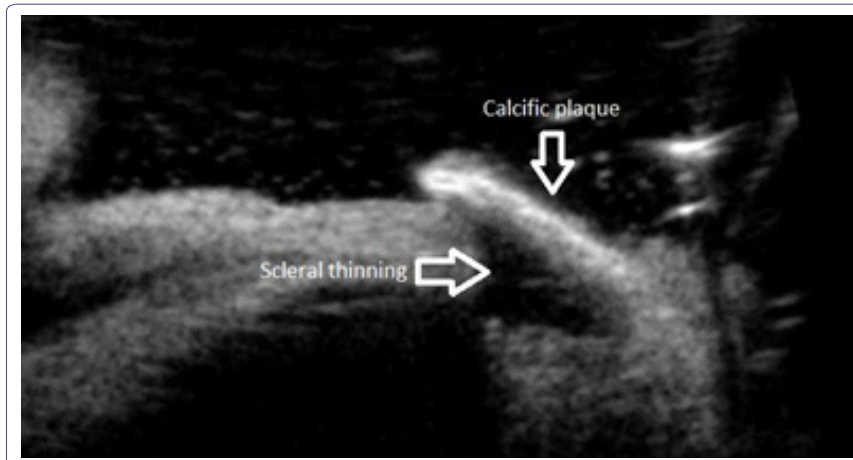

Figure 2: Ultrasonic biomicroscopy showing severe scleral thinning beneath the mass.

(Figure 3). An amniotic membrane (Ambiodry 2, IOP ophthalmics, Costa Mesa, CA) was sized to cover the scleral defect and secured with fibrin glue (TISSEEL, Baxter Healthcare Corporation, Deerfield, IL). Then, a scleral donor patch graft (Tutoplast, IOP ophthalmics, Costa Mesa, CA) was sutured over the amniotic membrane with interrupted 8-0 vicryl. Conjunctiva from the peritomy was pulled over the scleral patch. Another slightly thicker amniotic membrane (Ambio 5, IOP ophthalmics, Costa Mesa, CA) was glued over the conjunctiva to protect and provide further support for the scleral patch graft because the conjunctiva was very thin and inflamed. Finally, a large diameter contact lens (Kontur, Hercules, CA) was used to cover the entire corneal surface as well as the graft.

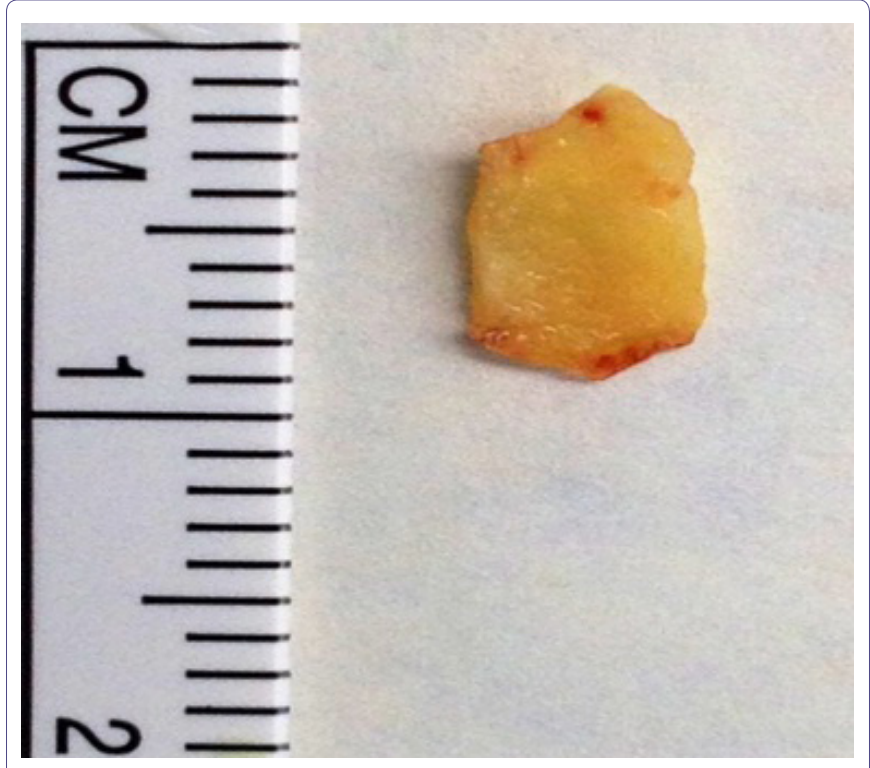

Figure 3: Senile calcific plaque measuring approximately $8 \mathrm{~mm}$ in size.

Postoperatively, histopathologic examination of the excised tissue specimen was reported as a senile calcific plaque. The patient was treated with topical ofloxacin and pred forte 4 times daily and an oral prednisone taper. After 6 weeks, the amniotic membrane had been absorbed, the graft was stable, the IOP had been raised to $8 \mathrm{mmHg}$, and the anterior chamber had been reformed. Nevertheless, she had progressive scleral thinning extending superonasally from the scleral patch graft. Oral prednisone was increased from 20 to $60 \mathrm{mg}$ daily. After 3 months, the prednisone was stopped. Her pain had completely subsided and the scleral thinning was no longer progressing. The best corrected visual acuity was 20/100 at 9 months follow-up. 


\section{Discussion}

This case represents a rarely reported complication of senile calcific plaques. Two previous cases of scleral perforation associated with a dehisced senile calcific plaque have been reported. One of these resulted in enucleation $[3,4]$. This may be the first report of dehiscence of a senile calcific plaque in the presence of perforation from necrotizing scleritis.

A variety of options for surgical management of scleral perforation associated with necrotizing scleritis exist including patch grafting with fascia lata, processed pericardium, dermal, and scleral patch grafts [6-9]. In this case, multiple layers of different materials were used including a scleral donor patch graft secured in place between two amniotic membranes with the thicker membrane on the outside The presence of necrotizing scleritis made this case exceptionally difficult to manage and enucleation was considered due to the patient's severe eye pain and poor prognosis. But ultimately, the arrangement of a scleral donor patch graft sandwiched between layers of amniotic membrane has held up well over time and prevented her from losing her eye.

In conclusion, necrotizing scleritis may rarely be associated with dehiscence of a senile calcific plaque and perforation of the already thinned sclera deep to the plaque. These cases may be effectively managed using a combination of scleral patch grafting with amniotic membrane transplant.

All authors certify that they have NO affiliations with or involvement in any organization or entity with any financial interest (such as honoraria; educational grants; participation in speakers' bureaus; membership, employment, consultancies, stock ownership, or other equity interest; and expert testimony or patent-licensing arrangements), or non-financial interest (such as personal or professional relationships, affiliations, knowledge or beliefs) in the subject matter or materials discussed in this manuscript. The patient consented to the submission of a case report.

\section{References}

1. Reidy JJ (2012) External Disease and Cornea. American Academy of Ophthalmology, San Francisco, California, USA.

2. Rosa RH (2012) Ophthalmic Pathology and Intraocular Tumors. American Academy of Ophthalmology, San Francisco California, USA.

3. Manschot WA (1978) Senile scleral plaques and senile scleromalacia. Br J Ophthalmol 62: 376-380.

4. Lyall DA, Arran NH (2010) Scleral perforation secondary to a spontaneously dehisced scleral plaque: clinical features and management. Clin Experiment Ophthalmol 38: 533-534.

5. Watson PG, Hayreh SS (1976) Scleritis and episcleritis. Br J Ophthalmol 60: 163-191.

6. Zheng X, Kodama T, Goto T, Ohashi Y (2011) Autologous fascia lata grafts for scleral repair in eyes with infectious necrotizing scleritis. Arch Ophthalmol 129: $1225-1227$.

7. Lazzaro DR (2010) Repair of necrotizing scleritis in ulcerative colitis with processed pericardium and a Prokera amniotic membrane graft. Eye Contact Lens 36: 60-61.

8. Nguyen QD, Foster CS (1999) Scleral patch graft in the management of necrotizing scleritis. Int Ophthalmol Clin 39: 109-131.

9. Sangwan VS, Jain V, Gupta P (2007) Structural and functional outcome of scleral patch graft. Eye (Lond) 21: 930-935. 